\title{
Surgery of muscle adhesions and effects of multiple operations
}

\author{
EDWARD A. DUNLAP \\ Rhinebeck, New York
}

The management of strabismus complicated by adhesions is always difficult; surgery to eliminate adhesions usually creates fresh difficulties unless one of three new techniques (described in this paper) is used. Their use still provides no absolute guarantee of success, but clinical experience has proved their value to me. Even if multiple-stage surgery by the most skilful surgeons succeeds in correcting the strabismus (apart from undercorrecting, overcorrecting, or the occasional creation of a new imbalance), adhesions which impair function by mechanical restriction of movement are likely to be produced. Brown (personal communication) has stated that once adhesions occur they can never be fully eliminated and that their recurrence can not be fully prevented, but the risk may be mitigated by the techniques to be described.

Restricted ocular movement may be congenital or acquired, and may be mechanical or neurogenic in origin.

Mechanically acquired restrictions may be caused by:

(I) Adhesions;

(2) Contracture of the conjunctiva;

(3) Contracture of the antagonist of a paralysed muscle;

(4) Shortening or weakening of muscles by excessive resection or recession;

(5) Contracture or fibrosis of the muscle and/or the sheath.

Other possible causes are:

(I) Orbital fracture;

(2) Penetrating orbital injury;

(3) Surgery for retinal detachment;

(4) Thyroid ophthalmopathy.

Adhesions in their turn arise from:

(I) Rough, sloppy, slovenly surgical technique;

(2) Excessive or disregarded bleeding;

(3) Excessive cautery;

(4) Multiple procedures;

(5) Excessive tissue reaction;

(6) Suture reaction;

(7) Operative or postoperative infection.

Neurologically acquired restrictions arise from nerve paralysis, with or without secondary fibrosis in the involved muscle or secondary contracture of its antagonist.

\section{Acquired restrictions of ocular movement}

In my opinion adhesions, which are largely iatrogenic, cause most of these problems. The 
surgeon (or his predecessor) creates the mass and mess of adhesions by errors of surgical technique. The heavy-handed man who pulls, hauls, and tears tissue, who takes big bites with toothed forceps when small bites with smooth forceps will suffice, who disregards meticulous control of bleeding (or on the other hand uses excessive or heavy cautery), who wipes rather than sponges, who leaves talc or cotton in the field, who massages the belly of a muscle with a squint hook, who hangs a clamp on one muscle while exposing another, who stretches an already weakened or underacting muscle, who tears a capsule or fascial sheath rather than cutting it, who leaves long suture ends, who closes the conjunctiva with heavy suture material and large bites, and who disregards in any other way a gentleness with and respect for tissue, such a man asks for complications-and deserves them. There are, however, certain patients with an innate tendency to manufacture excessive scar tissue in response to sutures or foreign bodics.

Bleeding may also cause adhesions; only Ingram (1966) does not agree with this idea. The best preventative is the instillation of a few drops of $\mathrm{I}: \mathrm{I}, 000$ adrenaline before the operation and occasionally during surgery if required.

Adhesions may occur at various sites:

(I) Between the muscle belly or its tendon and the globe;

(2) Between the muscle or capsule and the overlying conjunctiva;

(3) Between the sclera or intermuscular tissue and the conjunctiva.

Also diffuse sheets of scar tissue may involve large areas of muscle, capsule, intermuscular tissue, and conjunctiva, causing a fused mass.

The examination and diagnosis of restricted ocular movement are not included in this paper, but forced (passive) duction tests should always be performed under local anaesthesia in adults and cooperative children, or under general anaesthesia in children up to ro years of age. The use of general anaesthesia is justified by the value of the information so obtained. It is better to do this preliminary duction test as a separate procedure in order to plan the subsequent surgery. The mobility of the eye, both into and away from the field of action of the muscle being tested, should be carefully ascertained; a fibrotic muscle can restrict movement in either direction or both. The forced duction test should be repeated during the actual surgical procedure to verify that the restriction has been eliminated. Failure to achieve full, free passive movement of the globe at the end of surgery implies limited success or even failure of the operation, because the restricting tissue has been incompletely excised During the actual operation, the excision of all scar tissue or excess tissue around and between the muscles is imperative, but this is sometimes a formidable procedure.

\section{Methods of avoiding adhesions}

The three surgical techniques of proven value are:

(I) Implantation of the plastic material Supramid Extra in sheet, sleeve, or cap form, depending on the area to be covered;

(2) Conjunctival relaxation by recession (also termed bare sclera closure);

(3) Temporary anchoring sutures from the sclera through the eye lids to the external skin.

(1) Implantation of plastic material

This is directed both at relief of existent adhesions and at prevention of new or recurrent ones. The principle is to place an inert sheet of material as a barrier between tissues where adhesions have occurred or may be expected to develop. 


\section{(a) SU P R A I ID}

A detailed study of implanted materials (Dunlap, 1967) has shown Supramid Extra to be the best material for this purpose, being far superior to Gelfilm. The Supramid is available in three forms: sheets, seamless sleeves in diameters of 4,5 , and $6 \mathrm{~mm}$., and curved $\frac{1}{8}$ or $\frac{1}{4}$ spheres or caps. The material is inert, well tolerated, non-antigenic, thin $(0.05 \mathrm{~mm}$.), easily placed, and permanent. Moreover, repeated sterilization by autoclaving does not alter its structure.

Because most adhesions are relatively widespread, the sheet form is most commonly used. It is usually placed between muscle and globe or between conjunctiva and underlying tissue, and it must be sutured in place, 8-o silk or any synthetic suture being acceptable. Each corner, especially the two posterior ones, must be tacked down or the material may creep forward. The sharp corners should be trimmed to stop them cutting into the conjunctiva. Closure of the conjunctiva over the implant must be tight to prevent the formation of a fistula or the extrusion of the edge of the sheet. In placing it under a disinserted muscle that is to be re-attached, care should be taken not to place the Supramid within I mm. of the new attachment-its incorporation into a re-attachment may prevent proper union between muscle and sclera.

When a very large area is to be covered (a quarter or half of the globe), attachment of the posterior edge of the material to the sclera can be technically difficult, and the procedure is further complicated by the buckling that occurs when a flat sheet is fitted to a curved surface. This led Knapp (personal communication) to devise a curved sheet to fit the contour of the globe. These curved pieces of plastic are termed caps.

In the rare situation in which muscle only is involved, the intermuscular zones are clean, and the muscle needs protection both above and beneath, the sleeve type of insert may be used. This is placed by sliding it over the belly of a detached muscle for the desired length; the newly placed suture is slipped through the sleeve, and then the muscle is pulled through the sleeve or the sleeve is pulled down over the muscle. Sleeves need not be sutured in place. The original sleeves, which were seamed, tore open easily but this problem has been eliminated by the manufacture of seamless sleeves. No sleeve at present available is large enough to encase the muscle and its capsule together, but if required a sheet can be wrapped round and its edges sutured together. In the operation of advancing the inferior oblique, it is a simple matter to slip a sleeve over the muscle to protect it from adhering to the lateral rectus.

Histologicai ztudies of the behaviour of Supramid, reported in my original paper (Dunlap, 1967), have shown that tissue acceptance is excellent and that, even when large areas are covered, the reaction is no greater than in similar situations where no foreign material is intrs, auced.

To the best of my knowledge, I have had only two complications:

(i) One of the early-placed sleeves was too large and it tented the overlying conjunctiva to give a noticearle bulge. This sleeve was removed and a smaller one placed without further complication.

(ii) A haemorrhage occurred inside a sleeve approximately 2 years after its insertion. The patient con!plained of the sudden appearance of an elevated bluish mass over the lateral rectus muscle. Examination showed what appeared to be eroded sclera and exposed choroid, but on exploration the sclera was found to be intact and the bluish mass to be inside the plastic sleeve. When the sleeve was opened, blcod and some necrotic tissue were evacuated. Healing was uneventful without restriction of movement.

Knapp (personal communication), who has had much more experience with Supramid, has encountered no serious complications. 
No control studies are feasible because of the wide variety of surgical techniques involved.

(b) BUTYL GYANOAGRYLATE ADHESIVE

Dunlap, Dunn, and Rossomondo (I969) and Dunlap (I97I) suggested the paradoxical use of an adhesive to prevent adhesions. When the liquid polymer is painted onto a surface, immediate polymerization forms a plastic film, plaque, or sheet which is just as inert and impenetrable as the Supramid film. It is sometimes preferable to Supramid sheet, particularly when the conjunctiva in front of a recessed muscle is apt to adhere to the sclera. A film of the liquid is painted on to sclera dried by sponging (moisture triggers polymerization), using a siliconized iris repositor or a spatula fashioned from any plastic. The liquid changes in I or 2 seconds to a somewhat rough-surfaced plaque or sheet. The edges may be trimmed and any material inadvertantly placed on neighbouring tissue can be removed. The fact that the surface is relatively rough as compared with Supramid does not impair the movement of tissue over it. The plaque does not require to be sutured, but conjunctival closure must be exceptionally tight to avoid the formation of a fistula. If a fistula does occur, the acrylic material must be removed (it comes out in bits and pieces with a plain or toothed forceps), the edges of the conjunctival opening must be freshened, and the opening resutured. This material, manufactured in the United States by Ethicon and named Coapt Tissue Adhesive, is not yet available there commercially because of federal restrictions. It is however available in Germany and is marketed there under the name of Bucrylate.*

\section{(2) Conjunctival recession (bare sclera closure)}

Perhaps first described by Boeckmann ( 1897 ), redefined by Cole (1965), and popularized by Knapp (197I), the principle of this technique is to release the shrunken conjunctiva which may develop at the site of contracted muscle. A taut conjunctiva can be recognized by the stretch-lines and furrows which occur when the preoperative forced duction test is performed. Recession of the conjunctiva is easily achieved by incising the conjunctiva at the limbus, undermining it over the desired area, and then re-attaching it to the sclera by a few sutures further back. The bared sclera that is left exposed heals by the formation of granulation tissue. Sometimes a piece of the conjunctiva has to be excised and discarded. The original diagram shown by Cole ( 1965 ) is now an illustration of how not to do it, in that:

(i) No conjunctival lip is left between limbus and bare sclera;

(ii) Only a few sutures are required to tack down the new edge;

(iii) The recession shown is too small and would be inadequate in most cases.

Recessions of conjunctiva which leave the muscle exposed have been performed without untoward incident, but I would not recommend this procedure.

\section{(3) Temporary anchoring sutures}

First reported by Gruening (I89I) and later by Braun (1958), though Callahan (I96I) receives most of the credit nowadays, the principle is to hold the eye in the desired postoperative position for 5 to 7 days until healing is well advanced and any new formation of scar tissue will tend to keep the eye in that position. The anchoring is effected by sutures inserted into the sclera, brought out through the eyelid, and tied over rubber, cotton, or oiled-silk pegs. A relatively heavy silk or synthetic suture is required to minimize the risk of cutting throught the sclera. The scleral bite can be adjacent to the limbus or may be made 
posteriorly in the muscle stump; placement in the limbus usually allows less pull. The suture may be brought out through either lid, usually the upper one. It is advisable to bring the suture out through the tarsal plate to reduce the possibility of its cutting through the skin. If this happens traction on the eye will be lost. I have found that if I overcorrect by $5^{\circ}$ the globe will finally come to rest in the desired position.

The only complication that I have encountered is that if a suture cuts through the sclera it leaves a residual grey line of choroid showing under the scar. Some weakening of traction strength after a day or two is not uncommon and this in turn tends to release the globe from its anchorage. A corneal erosion which can be serious will occur if the suture is permitted to cross over the cornea on its way out through the lid.

This technique is also valuable in surgery of the tendon sheath syndrome, for which a specialized method of anchoring has been described by Scott and Knapp (1972).

In addition to these three surgical procedures, the choice of suture material merits attention. With multiple operations sensitization to catgut may develop, and this may increase postoperative reaction. I changed to the routine use of synthetic sutures (Supramid Extra) 2 years ago, since when I have noted a reduction in postoperative reaction even in uncomplicated cases. The use of synthetic material is even more beneficial in multiple operations. The surgeon's former reluctance to use synthetic material because of its permanency is now invalid because absorbable synthetic material is now available. Dexon (manufactured by Davis and Geck), has a "life" of only 30 days. Its use in eye surgery is still prohibited by the federal government in the United States although it is permitted in general surgery. Parks and White (personal communication) will be reporting an experimental study in $14^{\circ}$ patients at the American Academy meeting in September, I973.

The routine use of steroids postoperatively is not common in squint surgery in the United States, but in complicated cases, in which increased reaction is anticipated, their use either topically or by local subconjunctival injection after surgery is recommended.

\section{Discussion}

FELLS How long do the sutures remain in place?

DUNLAP About 4 or 5 days. By 6 or 7 days they work loose anyhow, so that nothing is to be gained by leaving them longer.

I have used Supramid in at least fifty cases and sleeves in fifteen, and cyanoacrylate adhesive in about twenty cases. Knapp has used over roo Supramid sheets and caps and about a hundred sleeves. He reports that he has had no complications himself, but has seen some in cases sent to him from other surgeons, All these appeared to be due to improper use (sheet not sutured, conjunctiva not closed, and in one case a sleeve was placed not round the muscle but round a mass of garbage).

GILKES Operative bleeding should be avoided by a good surgical technique using pressure not cautery. However, if a pharmacological agent is required, then Otrivine has a much longer duration of action than Epipnephrine. Otrivine is used in ear, nose, and throat surgery and can be obtained as an ENT preparation. It lasts 5 to 6 hours, and has no systemic effects which need influence the choice of anaesthetic. I now use it for all ophthalmic surgical techniques.

SCOTT It is a mistake to lay too much emphasis on haemorrhage in isolation, because scar tissue is caused by inflammatory changes in tissue and not by haemorrhage alone. The bleeding is not as important as extreme respect for the tissue which you are handling, particularly the avoidance of anything which may give rise to acute inflammation. Retinal surgeons frequently damage extraocular muscle, but I find that the restriction of extraocular movement is rare. The number of ad- 
hesions seen when re-operating on detachment cases is often extreme; the muscles may stick to conjunctiva and to sclera but still cause little restriction of movement. What is it, therefore, about the adhesions in squint cases which causes the restriction of movement? Is it restriction between muscle and globe or between one muscle and another? How does one decide which muscle and which adhesions to operate upon?

DUNLAP I have examined the literature very closely. With the exception of Ingram (1966), all authors regard haemorrhage as a potent cause of fibrosis and adhesions, but $I$ agree that this is a subject which requires further research. Detachment surgeons seem to get away with very much more damage to muscles than the squint surgeon ever could. One of the factors is that, if the detachment surgery is successful and the patient can still see, his ability to fuse images well preoperatively often saves him from the effects of fibrosis. These minor complications would not be overcome in the strabismus patient who has very poor fusion, if any. In choosing to excise or not and in assessing where the adhesions are, I do not think that any precise rules can be given. I excise anything that looks as though it might be inhibiting the movement of conjunctiva, globe, or muscles.

HARCOURT The younger the patient the more the adhesions. Most of the patients seen by Mr. Scott have had their surgery at an older age, and have therefore produced fewer adhesions than those that come to the squint surgeon.

DUNLAP One of the reasons why young children produce a lot of scar tissue is that they have relatively thick episcleral tissue, yet there is no question but that many adults produce restricting adhesions, even though they have little episcleral tissue.

PARKS The "garbage" Dr. Dunlap referred to was actually fibro-fatty proliferative tissue replacement of Tenon's capsule resulting from the erroneous surgical technique of a "deep dipper". A "deep dipper" is a surgeon who dips his hook deeply into the incision as he attempts to pass it beneath the insertion of the muscle, and thus engages the external fat pad (which is normally contained within a very nice envelope) and brings this mass of tissue up into the incision. The fat is compressed by this manoeuvre, and the "deep dipper" then becomes a "fat popper", as he incises the envelope containing this fat. The fat comes out under pressure and ultimately forms the fibro-fatty proliferative tissue adhering to the sclera and muscle capsule. So the "deep dipper" who becomes a "fat popper" is the "garbage maker."

DUNLAP I should like to emphasize that Dexon sutures are ideal for squint surgery in that they are made of a compound sugar substance containing no protein which dissolves within 30 days. They are the only currently available absorbable synthetic suture, and have the great advantage that they eliminate the catgut reactions which often occur in multiple operations.

PARKS I agree that Dexon is a very satisfactory suture material, having greater tensile strength than catgut of equivalent size. The surface of the suture is not smooth, but appears to be composed of an infinite series of microrings. The suture's rough surface grabs at the tissue it passes over and this may require a slight modification of the surgeon's technique. The knot ties extremely well and remains tight, allowing the suture to be cut off close to the knot with no fear of its becoming untied. The sutures are electrostatic like human hair and tend to stick together. 